\title{
Treatment of Pediatric Venous Thromboembolism
}

\author{
Katie A Meier, MD ${ }^{1,2 *}$; Cristina Tarango, MD ${ }^{1,3}$
}

'Department of Pediatrics, University of Cincinnati College of Medicine, Cincinnati, Ohio, 2Division of Hospital Medicine, Cincinnati Children's Hospital Medical Center, Cincinnati, Ohio; ${ }^{3}$ Division of Hematology, Cincinnati Children's Hospital Medical Center, Cincinnati, Ohio.

GUIDELINE TITLE: American Society of Hematology 2018 Guidelines for the management of venous thromboembolism: treatment of pediatric venous thromboembolism

DEVELOPER: The American Society of Hematology multidisciplinary subcommittee
RELEASE DATE: November 27, 2018

FUNDING SOURCE: American Society of Hematology PRIOR VERSION: N/A

TARGET POPULATION: less than 18 years of age

\section{V} enous thromboembolism (VTE) occurs uncommonly in pediatrics, affecting 0.07-0.14 per 10,000 children. ${ }^{1,2}$ Yet, in the last 20 years, the incidence of VTE in hospitalized children has increased dramatically to approximately 58 per 10,000 admissions. ${ }^{3}$ This increase may be attributed to improved survival of very ill children, better diagnostic imaging modalities, and heightened awareness by managing physicians. ${ }^{3}$ Randomized controlled trials are lacking in pediatric thrombosis, and clinical care is based on extrapolation of adult data and expert consensus guidelines. ${ }^{4,5}$ In 2014, the American Society of Hematology (ASH) sought to develop comprehensive guidelines on thrombosis. The pediatric VTE treatment guideline is one of six published to date.

\section{RECOMMENDATIONS FOR THE HOSPITALIST}

The following are five selected guideline recommendations thought most relevant to pediatric hospitalists. Three focus on the central venous access device (CVAD), since it is the most common risk factor for pediatric VTE. ${ }^{1}$ Recommendations were graded as "strong" if most providers, patients, and policy makers agreed with the intervention and if it was supported by credible research. Conditional recommendations had less uniform agreement with an emphasis on individualized care and weighing patients' values and preferences. ${ }^{6}$

Recommendation 1. It is recommended that pediatric patients receive anticoagulation, versus no anticoagulation, for symptomatic VTE (evidence quality: low certainty; recommendation strength: strong).

There is strong indirect data in adults that symptomatic VTE requires treatment, with limited direct evidence in children. As VTE occurs most commonly in ill, hospitalized children with the

*Corresponding Author: Katie Meier, MD; E-mail: katie.meier@cchmc.org; Telephone: 513-803-9177

Received: May 7, 2019; Accepted: May 9, 2019

( 2019 Society of Hospital Medicine DOI 10.12788/jhm.3246 potential for VTE to be life threatening, the benefit was felt to justify the strong recommendation despite low-quality evidence.

The primary benefit of anticoagulation in children with symptomatic VTE is the prevention of progressive or recurrent thrombosis with high morbidity and the prevention of life-threatening VTE. The greatest potential harm from the use of anticoagulation, particularly in very ill children, is the risk for major bleeding. ${ }^{4}$

Recommendation 2. Children with asymptomatic VTE can be managed with or without anticoagulation (evidence quality: poor; recommendation strength: conditional).

The panel focused on the unique features of pediatric VTE related to the heterogeneity in both the site and pathophysiology of VTE in children, such as age, presence of a CVAD, and comorbidities. There is little certainty that treating asymptomatic VTE is beneficial in the same way that treating symptomatic VTE would be in preventing recurrent thrombosis and embolization.

Until better evidence is available to guide care, the primary benefit of this recommendation is individualization of care related to each patient's risk-benefit profile and parental preferences.

Potential problems with using this recommendation include the cost of anticoagulant drugs and major bleeding if anticoagulation is used. Potential problems with not using anticoagulation would be progressive or recurrent thromboembolism. Close monitoring of children with VTE-regardless of whether anticoagulation is prescribed-is warranted.

\section{Pediatric Patients with Symptomatic CVAD-Related Thrombosis \\ Recommendations three through five pertain to CVAD-associ- ated thrombosis, so they are reviewed together.}

Recommendation 3. No removal of a functioning CVAD is suggested if venous access is still required (evidence quality: low certainty; recommendation strength: conditional).

Recommendation 4. It is recommended to remove a nonfunctioning or unneeded CVAD (evidence quality: low certainty; recommendation strength: strong). 
Recommendation 5. It is suggested to delay CVAD removal until after initiation of anticoagulation (days), rather than immediate removal if the CVAD is nonfunctioning or no longer needed (evidence quality: low certainty; recommendation strength: conditional).

CVAD is the most common precipitating factor for pediatric VTE, particularly in neonates and older children. ${ }^{1}$ Based on limited direct and indirect observational studies, there is low evidence of benefit for CVAD removal, but high-quality indirect evidence of harm and high cost, which the panel felt justified the strong recommendation for removing an unneeded or nonfunctioning line. If ongoing care can be safely administered without central access, removing the thrombosis stimulus is recommended. The guideline suggests keeping a functioning CVAD in a patient who requires ongoing venous access and placing high value on avoiding new line insertion when access sites may be limited to avoid the potential thrombogenic effect of new line placement.

In the limited direct and indirect observational studies identified, the optimal timing of CVAD removal is uncertain. Given the potential risk of emboli leading to pulmonary embolism or stroke, prior publications have suggested delaying removal until after three to five days of anticoagulation, particularly in children with known or potential right-to-left shunts. ${ }^{4}$ The risk of infection and bleeding with anticoagulation prior to CVAD removal was considered small by the panel. This recommendation is primarily based on the panel's anecdotal experience and first principles, which is a limitation.

\section{CRITIQUE}

Methods in Preparing Guideline. The panel included pediatric experts with clinical and research expertise in the guideline topic, including nine hematologists, one intensivist, one cardiologist, one hematology pharmacist, and one anticoagulation nurse practitioner. It also included two methodologists with evidence appraisal and guideline development expertise, as well as two patient representatives.

The panel brainstormed and prioritized questions to be addressed and selected outcomes of interest for each question. The McMaster University GRADE Centre vetted and retained researchers to conduct or update systematic evidence reviews and coordinate the guideline development using the Grading of Recommendations, Assessment, Development, and Evaluation (GRADE) approach. ${ }^{6}$ For each guideline question, the results of systematic reviews were summarized in GRADE Evidence-to-Decision tables. The evidence quality was categorized into four levels ranging from very low to high. For each recommendation developed, the panel agreed on the evidence quality, balance of benefits and harms of compared management options with consideration of resource use, and inferences regarding the potential associated values and preferences. The panel addressed 26 questions, which generated 30 recommendations.

Draft recommendations were made available online for review by stakeholders, including allied organizations, medical professionals, patients, and the public. Revisions were made to address pertinent submitted comments, but the recommendations were not changed. After approval by ASH, the guideline was subjected to peer review by Blood Advances.
Sources of Potential Conflict of Interest or Bias. The guideline was developed and funded by ASH. All participants' conflicts of interest were managed according to ASH policies based on recommendations of the Institute of Medicine and the Guideline International Network. A majority of the guideline panel had no conflicts. During deliberations, panelists with direct financial interests were recused from making judgments about relevant recommendations. The McMaster University-affiliated researchers had no conflicts.

Generalizability. While this guideline included 30 recommendations, the ones highlighted apply to the most commonly seen pediatric VTE cases in hospital medicine. ASH emphasized that these guidelines should not be construed as the standard of care, but as a guide to help clinicians make treatment decisions for children with VTE and to enable them to individualize care when needed. The greatest limitation of this guideline is the lack of strong direct supporting evidence in pediatric VTE management.

\section{AREAS IN NEED OF FUTURE STUDY}

Although there is increasing interest in pediatric VTE prevention and risk assessment, ${ }^{7}$ there is currently limited evidence on the best ways to mitigate VTE risk or anticoagulation-associated major bleeding in hospitalized children. The relatively low incidence of VTE in children makes large randomized controlled trials difficult, but several are ongoing. The Evaluation of the Duration of Therapy for Thrombosis in Children (Kids-DOTT) multicenter, randomized trial will inform care on the optimal duration of anticoagulation in children with a transient provoking factor, ${ }^{8}$ and several phase III studies are investigating the safety and efficacy of direct oral anticoagulants in children (NCT02234843, NCT02464969, NCT01895777, NCT02234843). These and future trials will better inform therapy in pediatric VTE.

Disclosures: The authors have no financial relationships or conflicts of interest relevant to this article to disclose.

Funding: No funding was secured for this study.

\section{References}

1. Andrew M, David M, Adams M, et al. Venous thromboembolic complications (VTE) in children: first analyses of the Canadian registry of VTE. Blood. 1994:83(5):1251-1257.

2. van Ommen $\mathrm{CH}$, Heijboer $\mathrm{H}$, Buller HR, Hirasing RA, Heijmans HS, Peters M. Venous thromboembolism in childhood: a prospective two-year registry in the Netherlands. J Pediatr. 2001;139(5):676-681. https://doi.org/10.1067/ mpd.2001.118192

3. Raffini L, Huang YS, Witmer C, Feudtner C. Dramatic increase in venous thromboembolism in children's hospitals in the United States from 2001 to 2007. Pediatrics. 2009:124(4):1001-1008. https://doi.org/10.1542/peds.2009-0768.

4. Monagle P, Chan AK, Goldenberg NA, et al. Antithrombotic therapy in neonates and children: antithrombotic therapy and prevention of thrombosis, 9th ed: American College of Chest Physicians Evidence-Based Clinical Practice Guidelines. Chest. 2012;141(2):e737S-e801S. https://doi.org/10.1378/chest.11-2308.

5. Monagle P, Cuello CA, Augustine C, et al. American Society of Hematology 2018 Guidelines for management of venous thromboembolism: treatment of pediatric venous thromboembolism. Blood Adv. 2018;2(22):3292-3316. https://doi.org/10.1182/bloodadvances.2018024786.

6. Guyatt GH, Oxman AD, Vist GE, et al. GRADE: an emerging consensus on rating quality of evidence and strength of recommendations. BMJ. 2008:336(7650):924-926. https://doi.org/10.1136/bmj.39489.470347.AD.

7. Faustino EV, Raffini LJ. Prevention of hospital-acquired venous thromboembolism in children: a review of published guidelines. Front Pediatr. 2017;5(9):1597-605. https://doi.org/10.3389/fped.2017.00009.

8. Goldenberg NA, Abshire T, Blatchford PJ, et al. Multicenter randomized controlled trial on Duration of Therapy for Thrombosis in Children and Young Adults (the Kids-DOTT trial): pilot/feasibility phase findings. J Thromb Haemost. 2015;13(9):1597-1605. https://doi.org/10.1111/jth.13038. 